\title{
Power Quality Disturbance Classification Based on A Novel Fourier Neural Network and Hyperbolic S-transform
}

\author{
Lin $\mathrm{Lin}^{1}$, Xiaohuan $\mathrm{Wu}^{2}$, Jiajin $\mathrm{Qi}^{2}$ and Hongxin $\mathrm{Ci}^{3}$ \\ ${ }^{1}$ College of Information and Control Engineering, Jilin Institute of Chemical \\ Technology, Jilin 132022, China \\ ${ }^{2}$ Municipal Power Supply Company of State Grid Zhejiang Electric Power \\ Company, Hangzhou 310007, China \\ ${ }^{3}$ Jilin Petrochemical Co information network data center, Jilin 132022, China \\ Corresponding author:.jllinlin@126.com
}

\begin{abstract}
Power quality $(P Q)$ disturbances recognition is the foundation of power quality analysis and improvement. In order to improve the classification accuracy and efficiency, a new classification approach based on modified Fourier neural networks (FNN) and Hyperbolic S-transform (HST) was designed for PQ disturbances classification. HST has better a time-frequency resolution than $S$-transform. The features extracted from HST results compose the input vectors of classifier. The DFP emendatory Quasi-Newton method is used to improve the learning ability of FNN and avoid local minimum problem. Three modified FNNs were used to construct a classifier with the structure of decision tree. Six types of disturbances with different noise ratio were simulated to test the classification ability of the new approach. Simulation results show that the new classifier has better classification accuracy than other classifiers based on BP neural networks and Fourier neural networks. The new approach is effective.
\end{abstract}

Keywords: power quality, power quality disturbance, Fourier neural network, DFP emendatory Quasi-Newton method, Hyperbolic S-transform

\section{Introduction}

Power quality (PQ) disturbances have different types and each type of these disturbances has a different course and effect to power system. Power quality disturbances recognition includes 2 steps: signal processing and pattern recognition [1]. The target of signal processing is extracting features from the original features to separate the different type of disturbance. The time-frequency analysis (TFA) methods, such as fast Fourier transform (FFT) [2], wavelet transform (WT) [3], Hilbert-Huang transform (HHT) [4] and S-transform (ST) [5], are usually used for processing the disturbance signals. FFT method could decompose the disturbances signals into different frequency and calculate the spectrum in each frequency. It is very effective for harmonic analysis. But FFT method could not locate the disturbance time and analyze the disturbances with short duration and wide frequency distribution such as voltage transient. The disturbances can be analyzed both from time and frequency area by WT, HHT and ST. Compared to other TFA analysis methods, ST has higher time-frequency resolution ratio and better noise immunity than WT and HHT. It is very satisfied for feature extraction of disturbances especially under high noise environment.

ST uses Gaussian window to analyze PQ disturbances with different time-frequency resolution in each different frequencies. The disturbances signals has different characteristic at different frequency area. The PQ disturbance signals change rapidly in the high frequency area and slowly in low frequency. Considering the time-frequency 
characters of PQ disturbance signals, the time-frequency window used to analyze PQ signals should narrow in the high frequency area to achieve the high time resolution and widen in the low frequency area to get the high frequency resolution, in order to get the best analysis result. The width of Gaussian window has a variable window width at different frequency which is inversely proportional to the frequency. It is very satisfied for PQ analysis.

But the width of window function of ST is constant if the frequency is established. The time-frequency feature presentation ability of ST can be improved by using some modified windows functions. Compared to other window function, the Hyperbolic window function used by Hyperbolic S-transform (HST) has wider width in the low frequency area and more narrow width in the high frequency area than traditional ST [6]. Therefore, the PQ disturbances feature presentation ability of HST is better than ST.

The typical pattern recognition methods, such as fuzzy rules (FR) [7], decision tree (DT) [8] and neural networks (NNs) [9-11], have been widely used for PQ disturbances classification. FR and DT methods for PQ disturbances recognition are very effective and easy to achieve. But the rules construction for FR and the thresholds for DT should be defined by the statistic of existing signal sets. The two methods cannot have adaptive adjustment for real random signal recognition. NNs have the characteristics of adaptive and robustness. The types of NNs commonly used for PQ disturbances recognition including Back Propagation Neural Networks (BPNN) [10] and Probabilistic Neural Networks (PNN) [11]. BPNN has been widely used in industrial area such as nonlinear analysis and pattern recognition. But the training method and structure parameters are difficult to identify. The limitations of learning ability and complexity of structure influence the accuracy and efficiency of the classification. PNN is very effective for PQ disturbance recognition with high classification accuracy and efficiency by the parallel training and classifying methods. But the space complexity is determined by the neuron number of input layer and hidden layer. A PNN based classifier needs a great deal of computer's internal storage if it has a large number of neurons in the input and hidden layers.

In this paper, a new classifier based on HST and Fourier Neural Network (FNN) is constructed to recognize 6 types of disturbances. Compared to other types of NNs, FNN has a simple structure and learning process. It is much more effective for PQ disturbances recognition than BPNN and less space complexity than PNN. The DFP emendatory Quasi-Newton method is used to improve the learning ability of Fourier Neural Network (FNN). The new learning method improves the learning ability of FNN. A classifier with tree structure based on 3 modified FNNs is designed to separate 6 types of disturbances. 3 features are extracted by HST. Each of the features is used as the input of a FNN. Each type of the disturbances signals is simulated with different signal noise ratio (SNR) and used for test the new classifier. Simulation results show that the modified FNN based classifier has better classification accuracy than other neural networks such as BPNN and traditional FNN.

\section{The Modified Fourier Neural Network}

The Fourier neural network is constructed on the theory of multilayer perception neural network and Fourier series. According to the theory of Fourier series, any nonlinear mapping which can be expanded into the form of Fourier series could be analyzed by FNN. FNN has better generalization performance and learning ability than BPNN, and it has great performance in classification. The FNN [12] is proposed in light of the Fourier series and neural network theory, which employs complex Fourier harmonic basis functions as its activation functions. The structure of FNN is a 3 layer includes input layer, hidden layer and output layer structure. Typically FNN has single input neuron in the input layer and multiple output neurons in the output layer. 


\subsection{The Theory of Fourier Neural Network}

According to the theory of the Fourier series, a periodic random function can expanded as a Fourier series. The coefficient of Fourier series will be tended to zero with the increase of the series number. Based on this principle, Fourier neural network can be established with the similar structure as BPNN. A typical structure of FNN is a three layer with single input and multiple outputs as Figure 1. The 3 layers include input layer, hidden layer and output layer. The weights between input and hidden layer are 1 . The weights between $d_{k}(k=1,2, \ldots, 2 n+1)$ and $x_{j}(j=1,2, \ldots, q)$ are $w_{k, j}$. $d_{k}(k=1,2, \ldots, 2 n+1)$ is the orthogonal function department generated by Fourier series which represent the different hidden neurons. They can be described as follows:

$d_{1}=1, d_{i+1}=\frac{2 \pi i t}{T}, d_{n+i+1}=\frac{2 \pi i t}{T}(i=1,2, \ldots, n)$

According to the weights definition and formula (1), the outputs of FNN are defined as follows:

$x_{j}(t)=w_{1, j}+w_{2, j} \cos \left(\frac{2 \pi t}{T}\right)+w_{n+2, j} \sin \left(\frac{2 \pi t}{T}\right)+\cdots+w_{n+1, j} \cos \left(\frac{2 \pi n t}{T}\right)+w_{2 n+1, j} \sin \left(\frac{2 \pi n t}{T}\right)$

(2)

Where $j=1,2, \ldots, q$. The formula (2) can be also described as:

$x_{j}(t)=\sum_{k=1}^{2 n+1} d_{k} w_{k, j}$

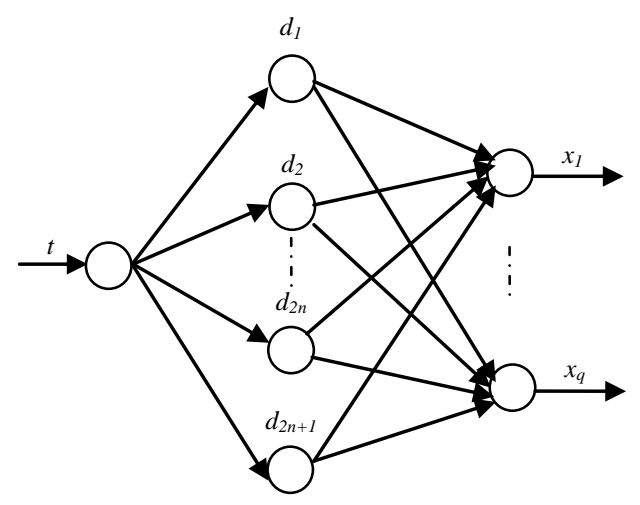

Figure 1. Structure of FNN

\subsection{The Improved Learning Algorithm based on DFP Emendatory Quasi-Newton Method}

The following formulas are defined as the output matrix, weights matrix and hidden matrix:

$O=\left[\begin{array}{c}O(1) \\ O(2) \\ \vdots \\ O(z)\end{array}\right]=\left[\begin{array}{cccc}O_{1}(1) & O_{2}(1) & \cdots & O_{q}(1) \\ O_{1}(2) & O_{2}(2) & \cdots & O_{q}(2) \\ \vdots & \vdots & \ddots & \vdots \\ O_{1}(z) & O_{2}(z) & \cdots & O_{q}(z)\end{array}\right]_{z \times q}$ 


$$
\begin{gathered}
W=\left[\begin{array}{cccc}
w_{1,1} & w_{1,2} & \cdots & w_{1, q} \\
w_{2,1} & w_{2,2} & \cdots & w_{2, q} \\
\vdots & \vdots & \ddots & \vdots \\
w_{2 n+1,1} & w_{2 n+1,2} & \cdots & w_{2 n+1, q}
\end{array}\right]_{(2 n+1) \times q} \\
D=\left[\begin{array}{c}
d^{T}(1) \\
d^{T}(2) \\
\vdots \\
d^{T}(z)
\end{array}\right]=\left[\begin{array}{cccc}
d_{1}(1) & d_{2}(1) & \cdots & d_{2 n+1}(1) \\
d_{1}(2) & d_{2}(2) & \cdots & d_{2 n+1}(2) \\
\vdots & \vdots & \ddots & \vdots \\
d_{1}(z) & d_{2}(z) & \cdots & d_{2 n+1}(z)
\end{array}\right]_{z \times(2 n+1)}
\end{gathered}
$$

The relationship between $\mathrm{O}, \mathrm{W}$ and $\mathrm{D}$ is define as $O=D W$, where $\mathrm{z}$ denotes the input vector $t_{z}$, and $\mathrm{O}$ is the actual output of FNN. Assume that the output of the FNN is:

$$
\begin{aligned}
& X=\left[\begin{array}{cccc}
x_{1}(1) & x_{2}(1) & \cdots & x_{q}(1) \\
x_{1}(2) & x_{2}(2) & \cdots & x_{q}(2) \\
\vdots & \vdots & \ddots & \vdots \\
x_{1}(z) & x_{2}(z) & \cdots & x_{q}(z)
\end{array}\right]_{z \times q} \\
& e_{j, r}=x_{j}(r)-O_{j}(r)
\end{aligned}
$$

Where $j=1,2, \ldots, q ; r=1,2, \ldots, z$. $\mathrm{r}$ delegate the input at $t_{r}$, the totally cost function is defined as:

$$
\zeta(W)=\frac{1}{2} \sum_{j=1}^{q} \sum_{r=1}^{z}\left(e_{j, r}\right)^{2}
$$

According to (6), the optimal model is defined as:

$$
\min \zeta(W)
$$

Then we calculate the optimal weight $W$. Traditional FNN uses steepest descent method and least square method to solve the problem of optimal model solution with the limitation of local extremism and lower convergence rate. The steepest descent induces the problems of local minimum and low learning velocity. The least square method with the limitation as follows: (1) if the model with colored noise, the estimation results of least square method is not unbiased and consistent; (2) least-square method is easy to fail to calculate the problems with high computational complexity. Compared to the existing training methods, Quasi-newton methods have a faster convergence speed and global convergence ability. They avoid the limitation of Newton method of complex computation cost of Hesse Matrix and become very effective searching algorithms. In the modern Quasi-newton methods, the DFP emendatory Quasi-Newton method has the advantages such as less computational complex, super linearity rate of convergence and global convergence. It is more suitable for solving the problem of learning search than traditional Quasi-Newton method.

In this paper, an improved Fourier neural network was constructed based on the DFP emendatory Quasi-Newton method to avoid the local minimum problem and has twoorder convergence velocity. 
We get the approximate function of $\zeta(W)$ by Taylor expansions near $W_{p}$, then approximate function is described as:

$\zeta(W) \approx \zeta\left(W_{p+1}\right)+g_{p+1}^{T}\left(W-W_{p+1}\right)+\frac{1}{2}\left(W-W_{p+1}\right)^{T} G_{p+1}\left(W-W_{p+1}\right)$

Where $G_{p}=\nabla^{2} \zeta\left(W_{p}\right)$, and $g_{p}=\nabla \zeta\left(W_{p}\right)$.

After calculate the derivation of $\mathrm{W}$ from both side of the formula (11), we have:

$g(W) \approx g_{p+1}+G_{p+1}\left(W-W_{p+1}\right)$

Let $W=W_{p}, s_{p}=W_{p+1}-W_{p}, \psi_{p}=g_{p+1}-g_{p}$, then

$G_{p+1}^{-1} \psi_{p} \approx s_{p}$

The approximate Hesse matrix is constructed by (13) is proposed like:

$H_{p+1} \psi_{p}=s_{p}$

Formula (14) is the quasi-newton conditions of Fourier neural network weights cost function. But the approximate solution of Hesse matrix in quasi-newton method is very difficult. In the new approach, the Hesse inverse matrix in Quasi-Newton method is solved by DFP correction formula. The algorithm is described as follows:

$u=s_{p}$

$v=H_{p} \psi_{p}$

$a=\frac{1}{s_{p} \psi_{p}}$

$b=\frac{-1}{\psi_{p}^{T} H_{p} \psi_{p}}$

Formula (15) to (18) satisfy the conditions as follows:

$a u^{T} \psi_{p}=1$

$b v^{T} \psi_{p}=-1$

The quasi-newton conditions described in (14) is satisfied by formula (15) to (20). Then the new function can be described as:

$H_{p} \psi_{p}+a u u^{T} \psi_{p}+b v v^{T} \psi_{p}=s_{p}$

According to DFP emendatory formula,

$H_{p+1}=H_{p}+a u u^{T}+b v v^{T}$

The inverse Hesse matrix can be modified by the update formula as follows:

$H_{p+1}=H_{p}+\frac{s_{p} s_{p}^{T}}{s_{p}^{T} \psi_{p}}-\frac{H_{p} \psi_{p} \psi_{p}^{T} H_{p}}{\psi_{p}^{T} H_{p} \psi_{p}}$ 
The learning algorithm based on DFP emendatory quasi-newton method of FNN is designed as follows:

Step 1: initialized the data, give the values of $W_{0}, H_{0}, 0<\varepsilon<1, p=0$.

Step 2: if $\left\|g_{p}\right\| \leq \varepsilon$, than stop learning; else, calculate $\vartheta_{p}=-H_{p} g_{p}$.

Step 3: make linear search according the direction of $\vartheta_{p}$, let

$$
W_{p+1}=W_{p}+\alpha_{p} \vartheta_{p}
$$

Step 4: update $H_{p}$ according to (15), and get $H_{p+1}$, let it satisfied (23).

Step 5: let $p=p+1$, then go to Step 2 .

\section{The Feature Extraction by HS-Transform}

PQ disturbances signals have different characteristics in different frequency area. The width of the window function should be narrow in the high frequency area to achieve higher time resolution and be wide in the low frequency area to get higher frequency resolution. C. R. Pinnegar [6] used a pseudo-Gaussian hyperbolic window instead of the Gaussian window and designed a new type of generalized S-transform which named Hyperbolic S-transform (HS-transform, HST). The generalized S-transform of a discrete time series $h(t)$ is derived by letting $\tau \rightarrow j T$ and $f \rightarrow n / N T$ is like:

$S\left(j T, \frac{n}{N T}\right)=\sum_{m=0}^{N-1} H\left[\frac{m+n}{N T}\right] G(m, n) e^{i 2 \pi m j / N}$

Where $G(m, n)=e^{-2 \pi^{2} m^{2} \alpha^{2} / n^{2}}, j, m, n=0,1, \ldots, N-1$.

Based on the DFT, The discrete inverse of HST is obtained as:

$h[k t]=\frac{1}{N} \sum_{n=0}^{N-1}\left\{\sum_{j=0}^{N-1} S\left[\frac{n}{N T}, j T\right]\right\} e^{i 2 \pi n k}$

Where $j$ and $n=0,1, \ldots, N-1$.

The Hyperbolic window $[2,17]$ instead of Gaussian window is designed as follows:

$$
\begin{aligned}
& w_{h y}=\frac{2|f|}{\sqrt{2 \pi\left(\alpha_{h y}+\beta_{h y}\right)}} \exp \left\{\frac{-f^{2} X^{2}}{2}\right\} \\
& X=\frac{\alpha_{h y}+\beta_{h y}}{2 \alpha_{h y} \beta_{h y}}(\tau-t-\xi)+\frac{\alpha_{h y}-\beta_{h y}}{2 \alpha_{h y} \beta_{h y}} \sqrt{(\tau-t-\xi)^{2}+\lambda_{h y}^{2}} \\
& 0<\alpha_{h y}<\beta_{h y}, \quad \xi=\frac{\sqrt{\left(\alpha_{h y}-\beta_{h y}\right)^{2} \lambda_{h y}^{2}}}{4 \alpha_{h y} \beta_{h y}}, \alpha_{h y} \text { is a forward-taper parameter, } \beta_{h y} \text { is a }
\end{aligned}
$$

backward-taper parameter, and $\lambda_{h y}$ is a positive curvature parameter which has units of time. Compared to the traditional ST, the HST has better time resolution ability in the high frequency area and better frequency resolution in the low frequency area. It is more suitable for power quality signal analysis.

Six types of disturbances including voltage sag, interruption, swell, flicker, harmonic and transient were simulated by Matlab 7.0 with different SNR and characters [8-11]. In order to compare the analysis ability of HST and ST, the analysis results of voltage sag 
with $0.1 \mathrm{pu}$ voltage dip and half period duration based on HST and ST are shown in Figure 2. We can see that the HST has better time-frequency feature representation than ST especially in the starting and ending points.
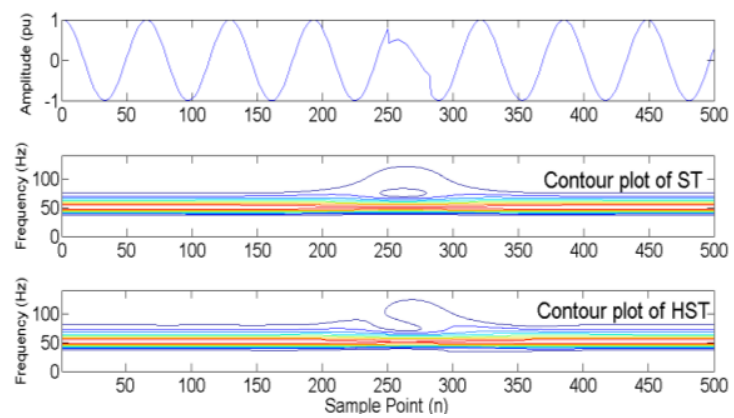

Figure 2. The Compare of Voltage Sag Analysis Results between ST and HST

Six types of disturbances signals were analyzed by HST, the results are shown in Figure 3 to Figure 8 . The first part of each figure is the original signal of disturbances. The second part of the figures is the contour plot of the results which is transformed by HST of each type of disturbance. The third part of the signal is the amplitude of fundamental frequency $(50 \mathrm{~Hz})$ at different sample point. The forth part of the figure is the mean amplitude of each frequency.
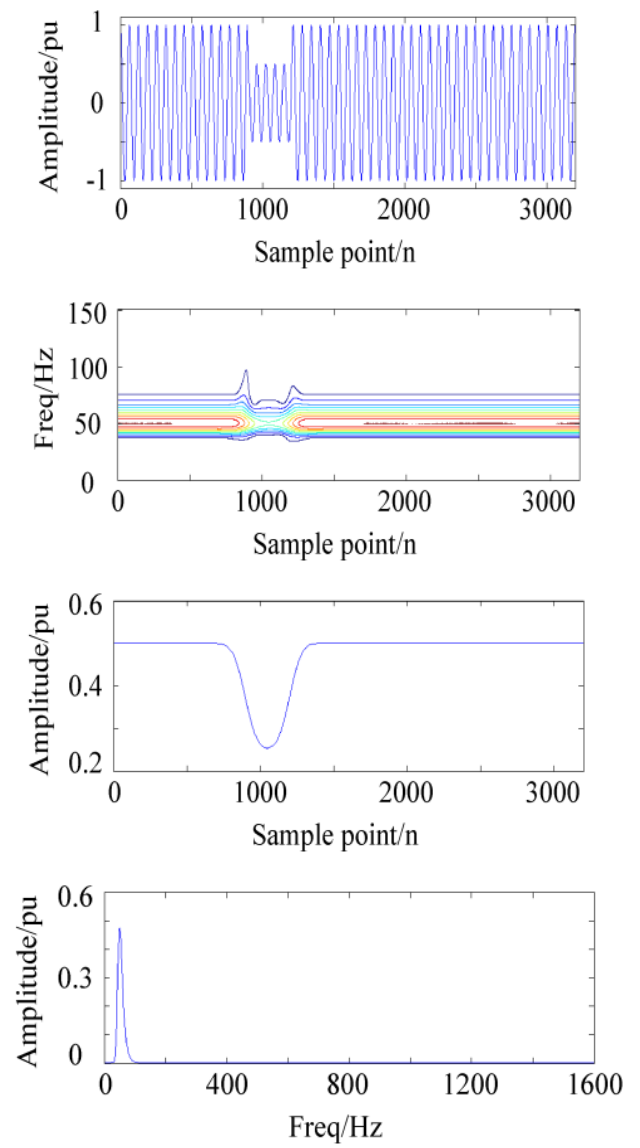

Figure 3. The Analysis of Voltage Sag Signal by HST 
International Journal of Signal Processing, Image Processing and Pattern Recognition Vol.9, No.1 (2016)
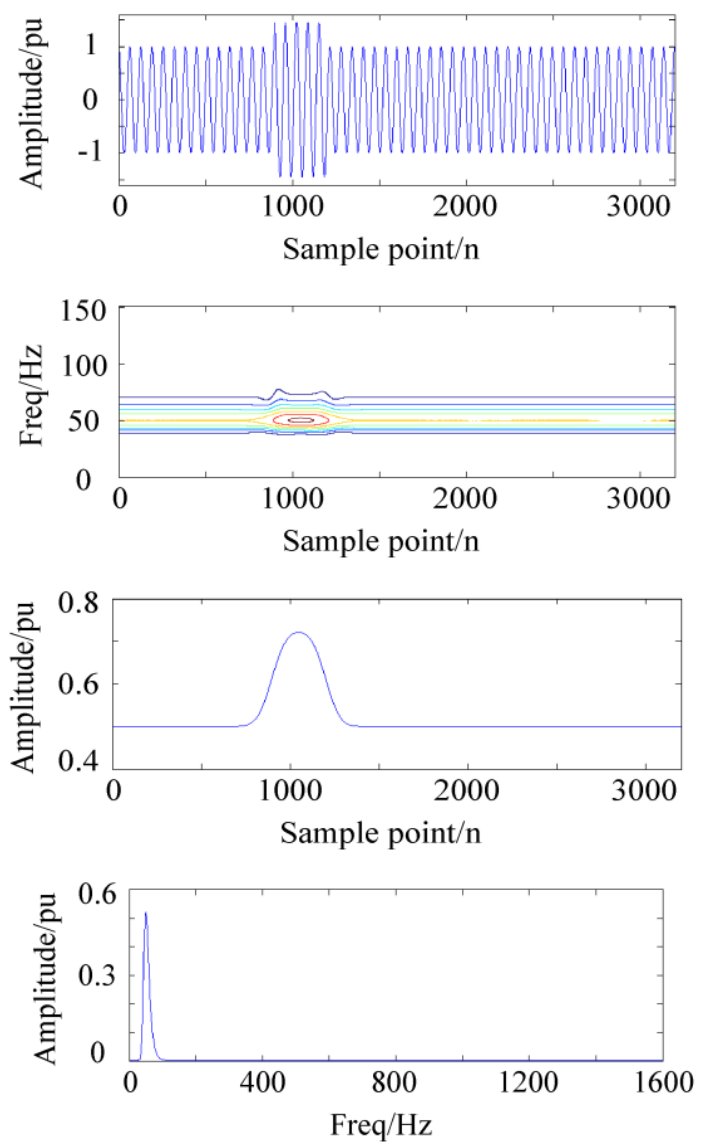

Figure 4. The Analysis of Voltage Swell Signal by HST
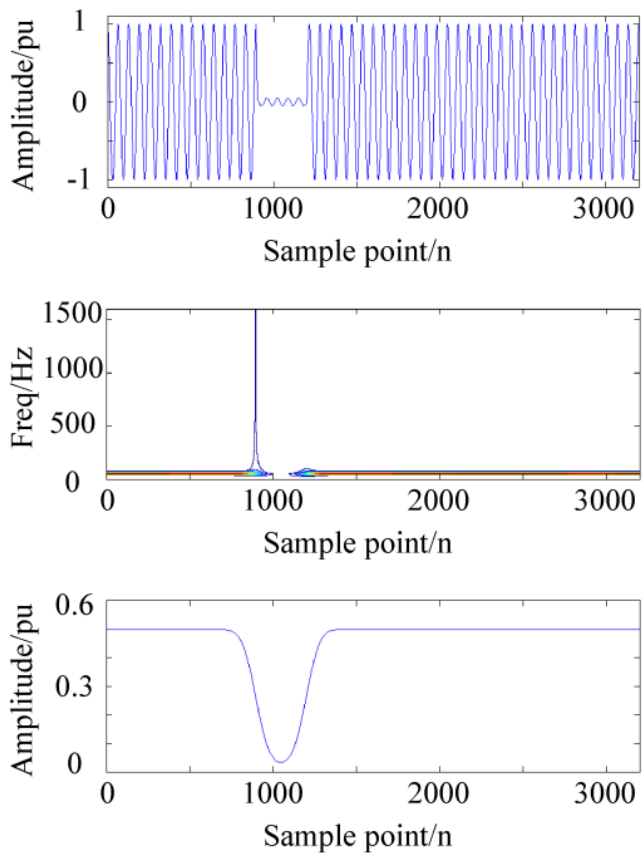


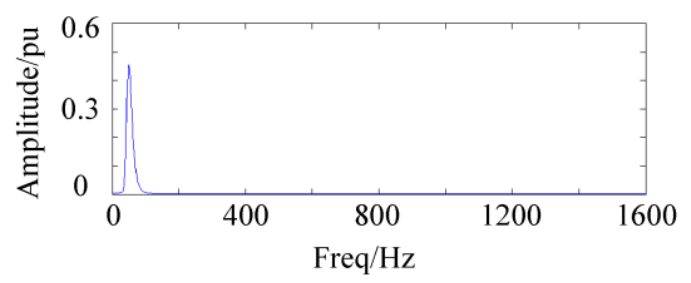

Figure 5. The Analysis of Voltage Interruption Signal by HST
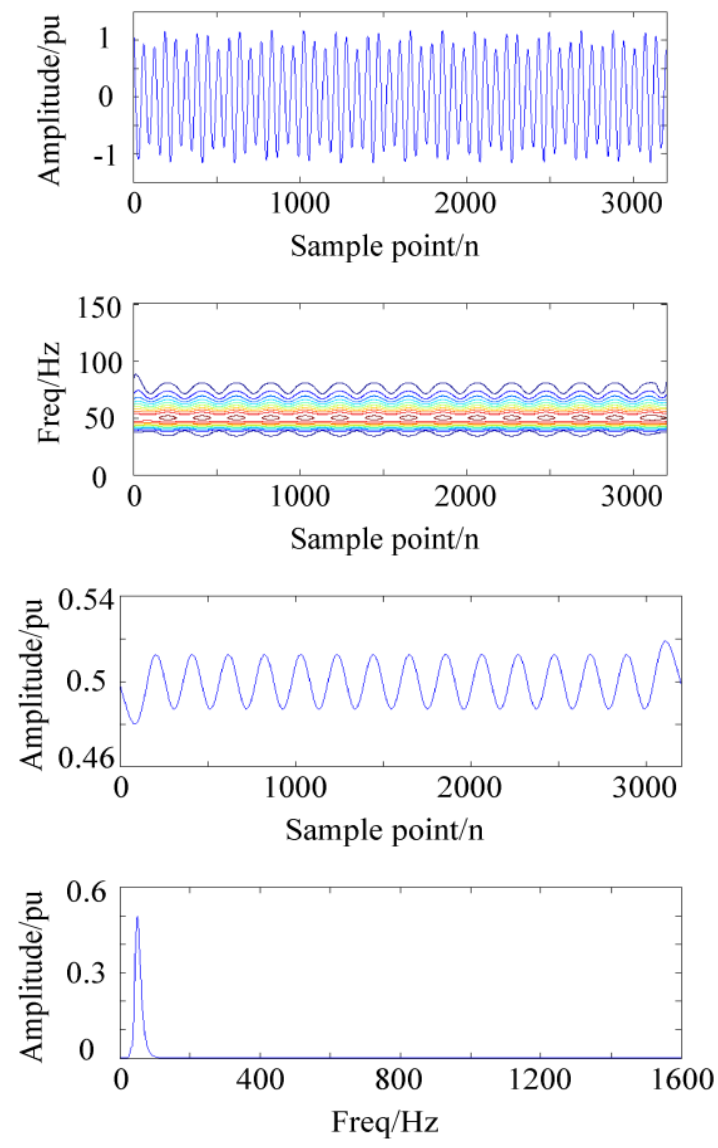

Figure 6. The Analysis of Voltage Flicker Signal by HST

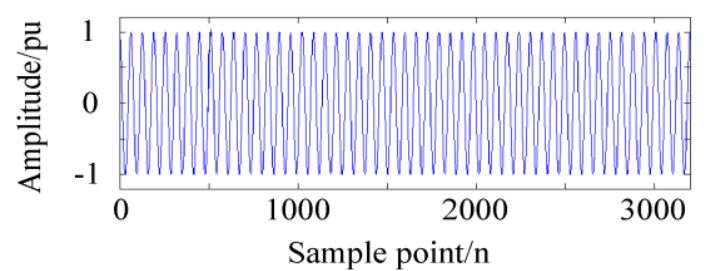



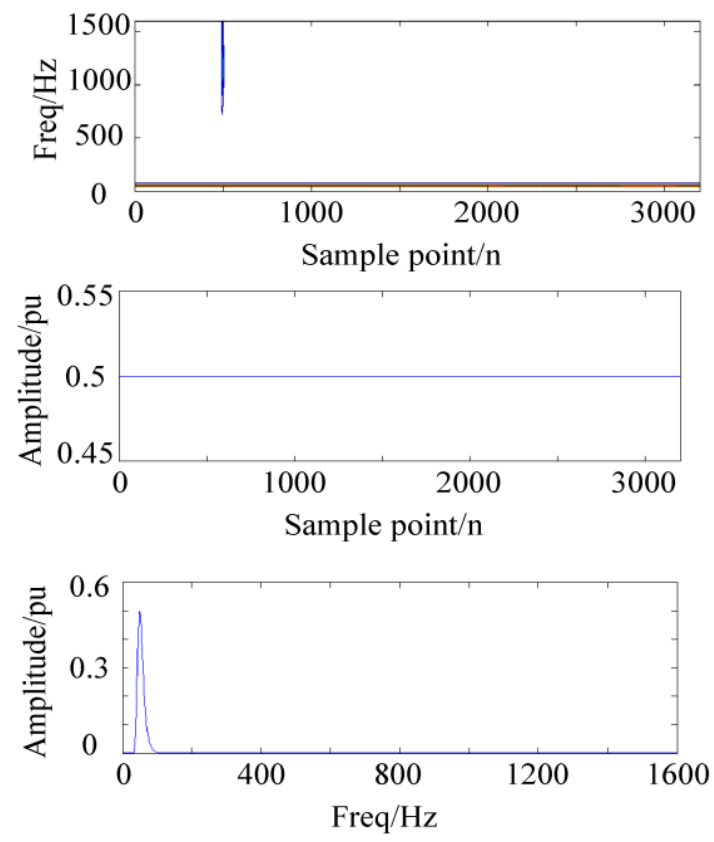

Figure 7. The Analysis of Voltage Transient Signal by HST
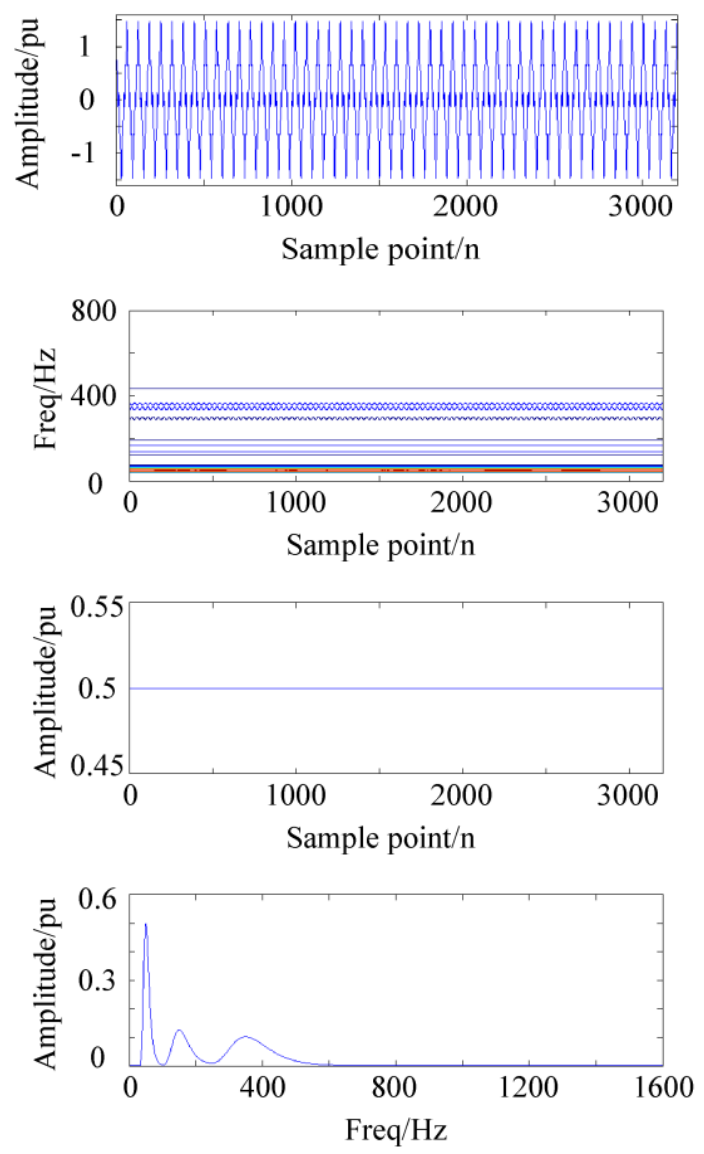

Figure 8. The Analysis of Harmonic Signal by HST

According to Figure 3 to Figure 8, the time-frequency characteristics of different types of disturbances can be described as follows. 
(1) The mainly energy of voltage sag, swell, interruption and flicker is focused near the fundamental frequency as part 2 shown, and the amplitude of fundamental frequency has obviously distortion as part 3 shown.

(2) Voltage flicker has a periodical distortion in the fundamental frequency; voltage sag, swell and interruption have distortion in the fundamental frequency when disturbances happen.

(3) The energy spectrum of harmonic has specific distribution in the times of harmonic and fundamental frequency. The distortion energy of voltage transient spreads in the high frequency area with short time duration.

So the disturbances can be recognized based on the features extracted from fundamental frequency and the frequency area. 3 features of disturbances were extracted from the results of HST as follows.

Feature 1: The amplitude factor $\left(A_{f}\right)$, the feature defines as $A_{f}=\frac{A_{\max }+A_{\min }-1}{2}$, where $A_{\max }$ is the maximum amplitude value of the signal simple and $A_{\min }$ is the minimum amplitude value of the signal simple. The value range of feature 1 is $0<A_{f}<1$.

Feature 2: The standard deviation ( $\left.\sigma_{S T D}\right)$ of the fundamental frequency $(50 \mathrm{~Hz})$.

Feature 3: The standard deviation of the mean amplitude of each frequency in the high frequency area (above $700 \mathrm{~Hz})$.

\section{The Structure of New Classifier}

Aim to separate 6 types of disturbances, new Fourier neural networks (NFNN) based on DFP emendatory Quasi-Newton method were used to construct the classifier with tree structure. The structure of the new classifier is shown in Figure 9:

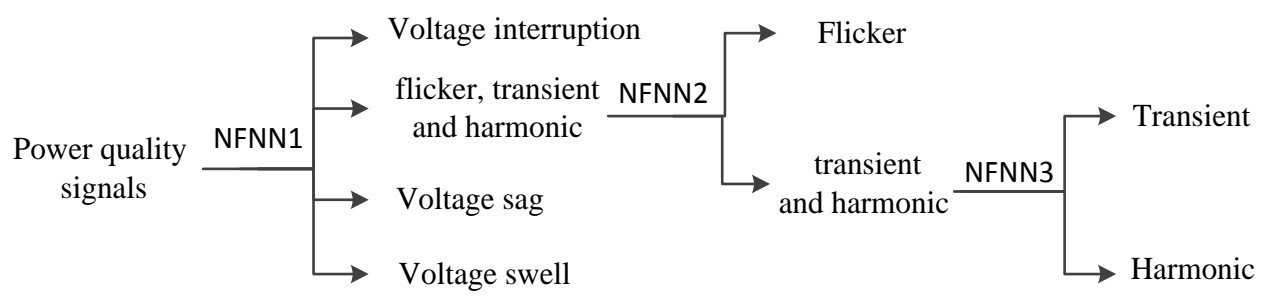

Figure 9. The Structure of the New Classifier

Feature1 is the input vector of NFNN 1. Voltage sag, swell and interruption are recognized by NFNN 1. Feature 2 is the input vector of NFNN 2. Voltage flicker is recognized in this step. Finally, feature 3 is used as the input vector of NFNN 3. Harmonic and voltage transient are recognized by NFNN 3. The neuron number of the hidden layer of each NFNN is 10.

Aim to test the classification ability of new classifier, 100 samples of each type of disturbance without noise were used to train the NNs. 100 samples of each type of disturbance with different SNR from $30 \mathrm{~dB}$ to $50 \mathrm{~dB}$ (signal noise ratio) and disturbances factors were used to test the classification ability of the new classifier. Other classifier based on BP neural networks (BPNN) and traditional Fourier neural networks (FNN) were also constructed to testify the improvements of the new classifier. The classification results of different classifiers were shown in TABLE 1. 
Table 1. Classification Results of Different Classifiers

\begin{tabular}{ccccc}
\hline \multirow{2}{*}{$\begin{array}{c}\text { Classification accuracy } \\
\text { type }\end{array}$} & HST and BPNN & HST and FNN & ST and NFNN & HST and NFNN \\
\cline { 2 - 5 } Sag & 97 & 99 & 95 & 100 \\
Swell & 96 & 99 & 95 & 100 \\
Interruption & 96 & 98 & 93 & 98 \\
Flicker & 97 & 100 & 98 & 100 \\
Voltage transient & 96 & 98 & 97 & 99 \\
Harmonic & 99 & 100 & 100 & 100 \\
\hline
\end{tabular}

The simulation result shows that the classifier based on NFNN has better classification accuracy than the classifiers based on BPNN and FNN. The new classifier also has better classification result than the classifier based on ST and NFNN.

\section{Conclusions}

This paper presents a new approach based on HST and NFNN to recognize 6 types of disturbances. The learning method of the FNN has improved by DFP emendatory QuasiNewton method. HST has better time-frequency resolution. The simulation results show that the new approach has better classification result than other methods for PQ disturbances with different characters. The new approach is effective and robustness.

\section{Acknowledgments}

This work is supported by the National Nature Science Foundation of China (Nos. 51307020), the Foundation of Jilin Technology Program (Nos. 20150520114JH) and the Science and Technology Plan Projects of Jilin City (Nos. 201464052).

\section{References}

[1] M. H. J. Bollen, P. Ribeiro, I. Y. H. Gu, and C. A. Duque, "Trends, Challenges and Opportunities in Power Quality Research", Euro. Trans. Electr. Power, vol. 6, no. 20, (2010).

[2] H. C. Lin, "Inter-Harmonic Identification Using Group-Harmonic Weighting Approach Based on the FFT", IEEE Transactions on Power Electronics, vol. 23, no. 3, (2008).

[3] M. H. Wang, and Y. F. Tseng, "A Novel Analytic Method of Power Quality Using Extension Genetic Algorithm and Wavelet Transform”, Expert Systems with Applications, no. 38, (2011).

[4] M. J. Afroni, D. Sutanto and D. Stirling, "Analysis of Nonstationary Power-Quality Waveforms Using Iterative Hilbert Huang Transform and SAX Algorithm”, IEEE Transactions on Power Delivery, vol. 28, no. 4, (2013).

[5] H. S. Behera, P. K. Dash and B. Biswal, "Power Quality Time Series Data Mining Using S-transform and Fuzzy Expert System”, Applied Soft Computing, no. 10, (2010).

[6] C. R. Pinnegar and L. Mansinha, "The S-transform with Windows of Arbitrary and Varying Shape", Geophysics, vol. 68, no. 1, (2003).

[7] S. K. Meher and A. K. Pradhan, "Fuzzy Classifiers for Power Quality Events Analysis", Electrical Power Systems Research, no. 80, (2010).

[8] M. Biswala and P. K. Dash, "Detection and Characterization of Multiple Power Quality Disturbances with a Fast S-transform and Decision Tree Based Classifier”, Digital Signal Processing no. 23, (2013).

[9] M. Oleskovicz, D. V. Coury, O. D. Felho, W. F. Usida, A. A. F. M. Carneiro and L. R. S. Pires, "Power Quality Analysis Applying a Hybrid Methodology with Wavelet Transforms and Neural Networks", Electrical Power and Energy Systems, no. 31, (2009).

[10] C. N. Bhende, S. Mishra and B. K. Panigrahi, "Detection and Classification of Power Quality Disturbances Using S-transform and Modular Neural Network", Electrical Power Systems Research, no. $78,(\mathbf{2 0 0 8})$

[11] N. T. Huang, D. G. Xu, X. S. Liu and L. Lin, "Power Quality Disturbances Classification Based on Stransform and Probabilistic Neural Network", Neurocomputing, vol. 98, (2012).

[12] W. Zuo and L. Cai, "A New Iterative Learning Controller Using Variable Structure Fourier Neural Network", IEEE Transactions on Systems, Man, and Cybernetics-Part B: Cybernetics, vol. 40, no. 2, (2010). 


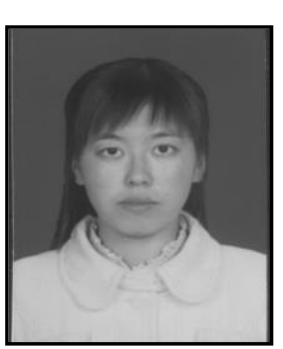

\section{Authors}

Lin Lin, She received her Master from Dalian University of Technology. She is currently an associate professor in college of information and control engineering, Jilin Institute of chemical technology. Her current research interests are focused on signal processing and pattern recognition.

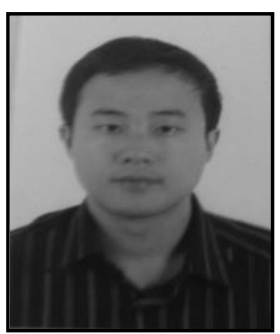

XiaoHuan Wu, He received his BS degree from Zhejiang University Of Finance \& Economics, China, in 2003 and received his MS degrees from Shanghai University of Finance \& Economics, China, in 2010. His main research interests are electric energy metering technology.

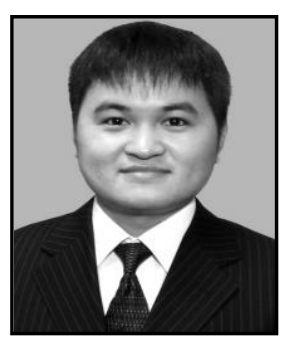

Jiajin Qi, He received his BS degree from Harbin Institute of Technology, China, in 2001 and received his MS degrees from Harbin Institute of Technology, China, in 2004. In 2009, He received his $\mathrm{PhD}$ degree from Harbin Institute of Technology, China. His main research interests are smart power consumption technology.

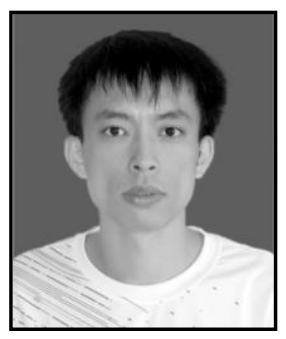

Hongxin Ci, He received the BS degree in School of Electrical Automation, Beihua University in 2000 . He is currently a deputy manager in data center of Jilin. His research interests are focused on compressive seensing and pattern recognition. 
International Journal of Signal Processing, Image Processing and Pattern Recognition Vol.9, No.1 (2016) 